\title{
Preoperative occlusal matrix aids the development of occlusal contour of posterior occlusal resin composite restorations - clinical rationale and technique
}

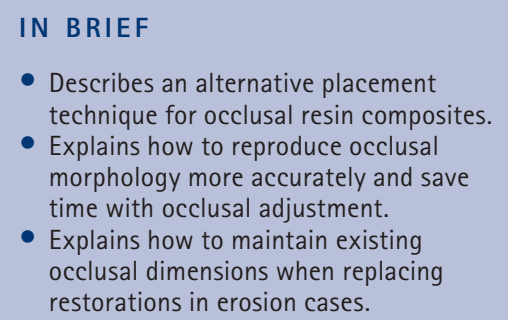

\author{
A. Geddes, ${ }^{1}$ J. Craig ${ }^{2}$ and R. G. Chadwick ${ }^{3}$
}

VERIFIABLE CPD PAPER

This clinical article describes and discusses a technique, using a preoperative occlusal matrix, whereby the occlusal morphology of a tooth may be replicated when placing a posterior resin composite. Two clinical cases are described. The technique offers the clinician considerable time saving for required occlusal adjustment is minimal.

\section{INTRODUCTION}

Concern has been expressed about the environmental impact of dental amalgam. Such interest has focussed upon the release of mercury into environmental water from the dental unit and also from the cremation of the deceased with amalgam restorations. ${ }^{1}$ The resultant environmental pollution that ensues has led many countries to ban dental amalgam. This has necessitated the use of alternative restorative materials of which resin composite is one. Such materials contain no mercury and are developments of the original chemistry, developed by R. L. Bowen, that consisted of the resin matrix 2,2-bis-[-4(2-hydroxy-3-methacryloyloxy-propyloxy)-phenyl-]-propane (commonly known as either BIS-GMA or Bowen's Resin) mixed with inorganic fillers, bonded to the matrix, by the coupling agent $\mathrm{Y}$-methacryloxypropyltrimethoxy silane. ${ }^{2-5}$ Modern derivatives of these early materials set by a polymerisation reaction that is initiated upon exposure of the material to visible blue light. Their

\footnotetext{
1,2Dental Foundation Trainee, ${ }^{{ }^{*}}$ Clinical Senior Lecturer and Honorary Consultant in Restorative Dentistry, The Dental School and Hospital, Park Place, Dundee, DD1 $4 \mathrm{HN}$

${ }^{*}$ Correspondence to: Dr R. G. Chadwick, The Dental School and Hospital, Park Place, Dundee, DD1 4HN Email: r.g.chadwick@dundee.ac.uk
}

\section{Refereed Paper}

Accepted 19 December 2008

DOI: $10.1038 /$ sj.bdj.2009.215

${ }^{\oplus}$ British Dental Journal 2009; 206: 315-317 clinical application, in the restoration of the posterior dentition, is widely taught in the dental schools of Ireland and the United Kingdom for they offer the advantages, when applied with acid etching and dentine bonding, of reducing the need to remove sound tooth substance for retention, good aesthetics, reinforcement of residual tooth structure and provide increased fracture resistance of the restored tooth. ${ }^{6}$ Their use also reduces future mercury environmental contamination. It is, however, acknowledged that with such a restorative material, the reestablishment of the occlusal morphology is both time consuming ${ }^{7}$ and dependent upon high skill. ${ }^{8}$ For this reason some have advocated the chairside fabrication of an occlusal matrix, before cavity preparation ensues, that may be used to contour the final resin composite restoration. ${ }^{7,8}$ The materials described for this purpose are either a clear polyvinyl siloxane bite registration material (Memosil, Heraeus Kulzer, Germany) $^{7}$ or a Vaseline separated temporary light-cured composite resin (Fermit, Vivadent, Brazil). ${ }^{8}$ Such a replication technique is, however, dependent upon either a virtually intact occlusal morphology, as displayed in the case of advanced hidden caries, ${ }^{9,10}$ or the presence of a failed restoration of the desired occlusal morphology. If these conditions are met, correct execution of the preoperative occlusal matrix technique, it is claimed, allows the final resin composite restoration to: (a) reproduce the original morphology and occlusion; (b) require no or minimal finishing and polishing; (c) reduce the incidence of voids at the occlusal surface; (d) minimise the amount of flash at the restoration margins; (e) provide an occlusal surface that is optimally polymerised due to the exclusion of air, by the matrix, during curing. ${ }^{7}$

Since 2000 all de novo posterior occlusal carious lesions presenting on the undergraduate restorative clinics in Dundee Dental Hospital and School, and warranting restoration, have been restored with directly placed and bonded resin composite. Where appropriate a variant of the pre-operative occlusal matrix technique has been employed. In addition, the technique has found application in the replacement of posterior restorations to replicate occlusal form where, due to toothwear, submargination of the restoration has occurred rendering it more time consuming to restore to the same occlusal scheme. Submargination arises when the natural tooth substance, surrounding a metallic intracoronal restoration, is eroded away upon chronic exposure to acid. This results in the restoration appearing proud relative to the surrounding tooth structure.

It is the purpose of this clinical technique paper to describe this method of restoration illustrated by a case treated 
by the first author whilst a final undergraduate student (Case One) and also to describe, as illustrated by a second case, how the basic technique may be modified to copy the occlusal morphology of a restoration where submargination has occurred due to toothwear.

\section{CASE STUDY ONE AND CLINICAL TECHNIQUE}

The patient was a 20-year-old female student who attended Dundee Dental Hospital having not visited a dentist since schooldays. She displayed, in many posterior teeth, the signs of occlusal caries despite a virtually intact occlusal surface. This caries was found to be extensive on bitewing radiographs. One such tooth was the upper left first permanent molar (Fig. 1). It was decided that as the patient had no amalgam restorations present, and the resultant cavity would be entirely bound by enamel, the restorative material of choice was resin composite. Prior to the commencement of cavity preparation local anaesthesia (lignocaine with 1:80000 adrenaline) was administered by buccal infiltration. Whilst waiting for this to take effect a preoperative occlusal matrix was formed using a transparent polyvinyl siloxane bite registration paste (Memosil). This was delivered from the manufacturers automix tip (Fig. 2) to the occusal surfaces of the opposing teeth and the patient invited to close upon it in centric occlusion. Once set the resultant registration and occlusal matrix (Fig. 3) was removed from the teeth and set to one side for both use at this visit and future ones. As other occlusal cavities, for restoration with resin composite, were planned for subsequent patient visits, the matrix spanned much of the dental arch. Thereafter the cavity was prepared using high and low speed burs with caries removal by both hand excavators and low speed round burs. The resultant cavity was lined with both the calcium hydroxide lining cement Alkaliner (3M ESPE, UK) and the light activated resin modified glass polyalkenoate Vitrebond (3M ESPE, USA). The enamel margins of the cavity were acid etched using a 37\% phosphoric acid etchant gel (Unogel, Unodent, UK) applied for

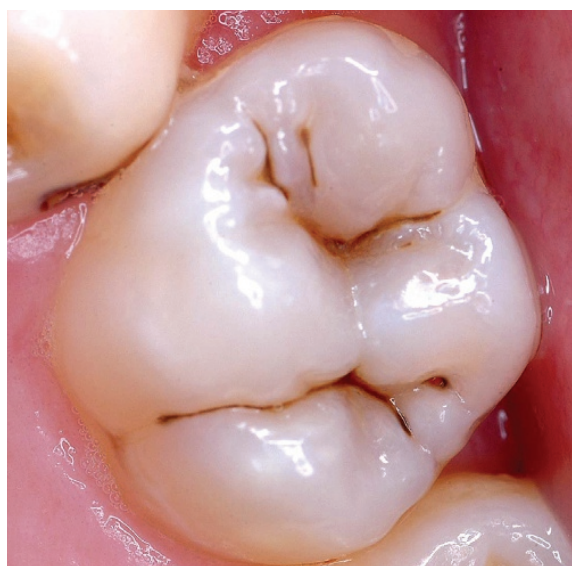

Fig. 1 Case One - The upper left first permanent molar prior to restoration

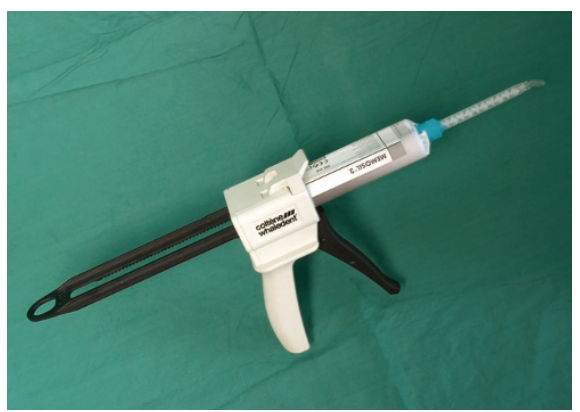

Fig. 2 Memosil in the automix delivery system

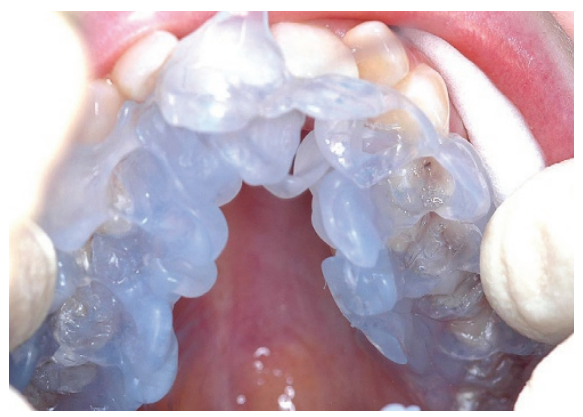

Fig. 3 Case One - The pre-operative Memosil occlusal matrix prior to removal and commencement of cavity preparation

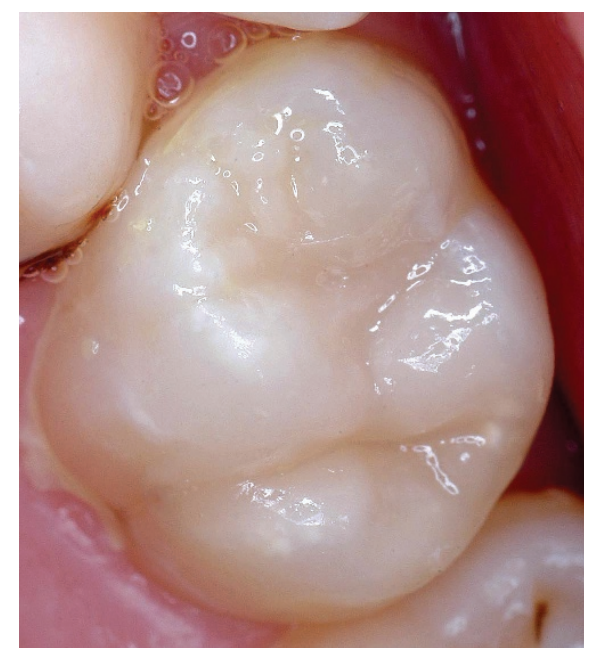

Fig. 4 - Case One - The completed occlusal resin composite restoration of the upper left first permanent molar

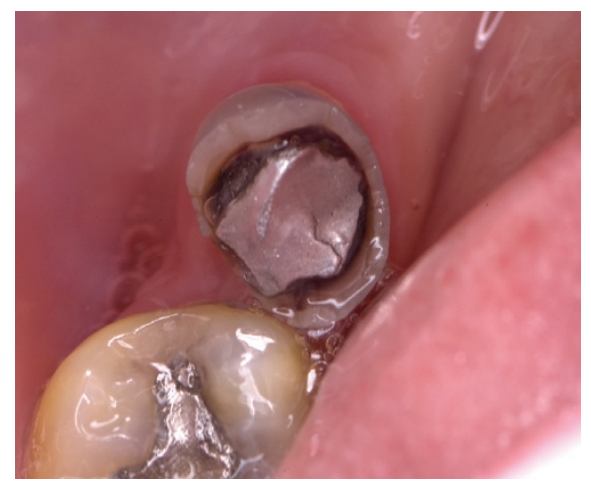

Fig. 5 Case Two - Failed DO amalgam in the lower left second premolar. This restoration displays marginal caries and the surrounding tooth substance has undergone submargination

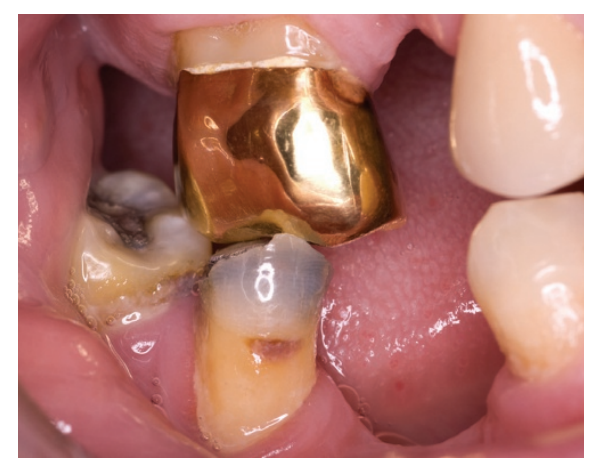

Fig. 6 Case Two - The lower left second premolar occludes with the gold crown, upper left first molar, and its occlusal morphology needs to be replicated to maintain the occlusal vertical dimension

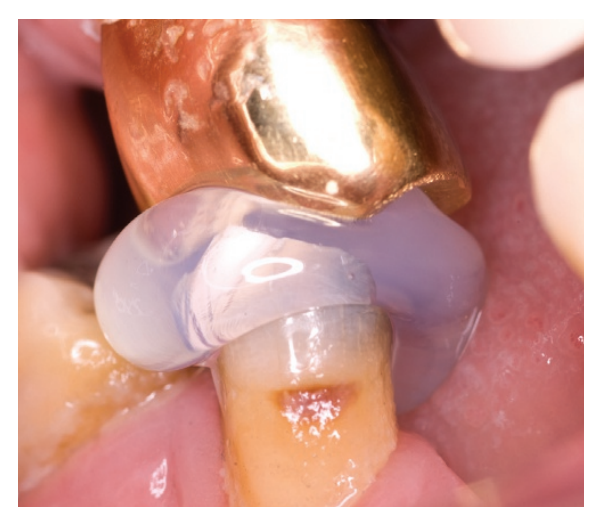

Fig. 7 Case Two - Forming the pre-operative Memosil matrix

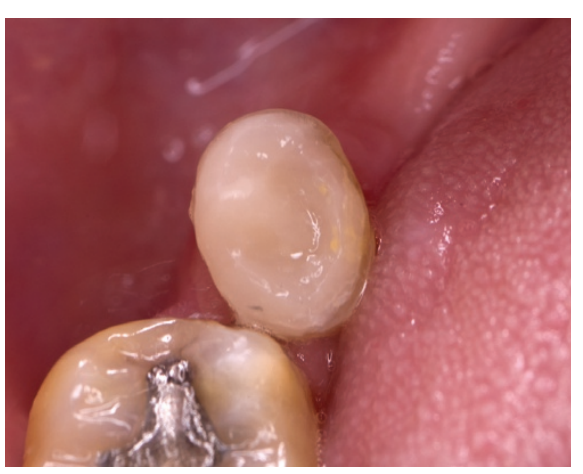

Fig. 8 Case Two - The completed restoration in the lower left second premolar 
30 seconds. The dentine bonding agent Clearfil SE (Kurray, Japan) was then applied, according to the manufacturer's instructions, to the walls of the cavity. The cavity was then incrementally filled with the nano filled resin composite Filtek Supreme (3M ESPE, USA) with each increment being cured for 30 seconds using a visible blue light curing unit (Smart Lite, DENTSPLY De Trey, Germany). Following the placement of the last increment the Memosil matrix was reseated on the teeth before being exposed to the curing unit. Once curing had been completed the occlusal matrix was removed and the occlusion of the final restoration checked. In this case no adjustments to restoration morphology were necessary but if required they could be made using composite finishing burs. Figure 4 shows the completed restoration.

\section{CASE STUDY TWO AND CLINICAL TECHNIQUE}

The patient was a 50-year-old male who was undergoing restorative treatment to address his toothwear. In the course of this it was noted that the disto occlusal amalgam in the lower second premolar had caries around its deficient occlusal margins with submargination of the surrounding tooth substance (Fig. 5). The tooth was strategic in maintaining the occlusal vertical dimension of the patient (Fig. 6) and as such, it was desirable that the replacement restoration should have the same occlusal scheme. In this case, prior to the formation of the preoperative Memosil matrix (Fig. 7), the marginal discrepancies of the existing restoration were temporarily made good with a conventional glass polyalkenoate cement (Fuji IX, GC, Japan). Thereafter the matrix was removed and both restoration and caries removal ensued. The resultant cavity was lined with Vitrebond and the margins of the cavity were acid etched and dentine bonded (Prime and Bond NT, Dentsply, UK) prior to restoration with resin composite (Spectrum, Dentsply, UK). The matrix was reseated following the insertion of the final increment of composite and then was light cured through the matrix. The cured restoration only required slight adjustment upon removal of the matrix (Fig. 8).

It should be noted that in all the reported cases rubber dam was not applied but scrupulous saliva ejector and cotton wool isolation was maintained throughout these procedures.

\section{DISCUSSION}

In our eight year experience of this technique the application of the pre-operative occlusal matrix, in the placement of posterior resin composites, reproduces the original tooth morphology and occlusion. Such restorations require no or minimal finishing and polishing. As great care, in the cases presented here, was taken not to greatly overfill the cavities, when placing the final increment of resin composite, the level of flash at the restoration margins was minimal. Such advantages of this technique have previously been reported by others. ${ }^{7}$ We further believe that the pressure applied by contact of the light curing tip upon the seated Memosil matrix, prior to curing the final increment of composite, prevented restoration overbuild. Although rubber dam was not applied in the cases presented here its application does not prevent use of the pre-operative occlusal matrix. In such an instance it is essential that matrix formation precedes both rubber dam application and cavity preparation so that centric occlusion may be captured in the matrix.

A novel application of the technique, as reported here, was to copy the occlusal morphology of a failed restoration that displayed submargination. The correction of deficiencies, using temporarily placed glass polyalkenoate cement, prior to the fabrication of the pre-operative matrix, enabled the patient's comfortable and familiar occlusal relationship and restoration morphology to be copied in the new corrected final restoration. Such application has not previously been reported.

As this paper is a case report we cannot verify the claimed beneficial effects of the technique upon the physical properties of the restoration. According to Hamilton et al. ${ }^{7}$ these are said to be:

- The exclusion of oxygen from the surface of the restoration during curing resulting in a surface that is optimally polymerised

- A reduction in voids in the final restoration, compared to a composite restoration placed conventionally. The presence of porosity within resin composite restorations has previously been shown to have adverse effects upon material dependability. ${ }^{11}$

Despite the argument that additional chairside time is required to make the pre-operative matrix, we and others ${ }^{7,8}$ have found that the time lost is more than compensated by the time gained by not having to contour the surface and make occlusal adjustments. It has been established by others ${ }^{12}$ that adjusting and finishing the occlusal surface of posterior resin composites, by conventional methods, to be in harmony with the opposing occlusion is very time consuming. The application of the preoperative occlusal matrix technique, as described here, obviates this need resulting in great time savings.

1. Arenholt-Bindslev D. Dental amalgam - environmental aspects. Adv Dent Res 1992; 6:125-130.

2. Bowen $R \mathrm{~L}$. Dental filling materials comprising silane treated fused silica, and a binder consisting of a reaction product of Bisphenol and Glycidyl Acrylate. 1962. US Patent Number 3066, 112.

3. Bowen R L. Properties of a silica reinforced polymer for dental restorations. J Am Dent Assoc 1963; 66: $57-64$

4. Bowen R L. Effects of particle shape and size distribution in a reinforced polymer. J Am Dent Assoc 1964: 69: 481-495.

5. Bowen R L. A method of preparing a monomer having phenoxy and methacrylate groups linked by hydroxyl glyceryl groups. US Patent Number 3179623. 1965.

6. Lynch C D, McConnell R J, Wilson N H F. Teaching of posterior composite restorations in undergraduate dental schools in Ireland and the United Kingdom. Eur J Dent Educ 2006; 10: 38-43.

7. Hamilton J C, Krestik K E, Dennison J B. Evaluation of custom occlusal matrix technique for posterior light-cured composites. Oper Dent 1998; 23: 303-307.

8. Duque $C$, Cristiana da Silva $R$, dos Santos-Pinto L. Treatment options for the occlusal surface of first permanent molars. J Clin Pediatr Dent 2004; 29: 5-10.

9. Ricketts D, Kidd E, Weerheijm K, de Soet H. Hidden caries: what is it? Does it exist? Does it matter? Int Dent J 1997: 47: 259-265.

10. Ricketts D N, Ekstrand KR, Kidd E A, Larsen T. Relating visual and radiographic ranked scoring systems for occlusal caries detection to histological and microbiological evidence. Oper Dent 2002; 27: 231-237.

11. Chadwick R G, McCabe J F, Walls A W G, Storer $R$. The effect of placement technique upon the compressive strength and porosity of a composite resin. J Dent 1989; 17: 230-233.

12. Dilley D C, Vann W F Jnr, Oldenburg T R, Crisp $\mathrm{R}$ M. Time required for placement of composite versus amalgam restorations. J Dent Child 1990; 57: 177-183. 